RICYDE. Revista Internacional de Ciencias del Deporte doi: $10.5232 /$ ricyde

Rev. int. cienc. deporte

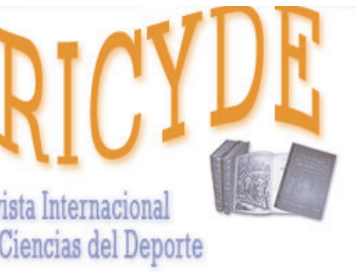

RICYDE. Revista Internacional de Ciencias del Deporte VOLUMEN XI - AÑO XI

Páginas:173-185 ISSN:1885-3137 No 40 - Abril - 2015

\title{
Análisis de la condición física de jugadores nacionales de baloncesto en silla atendiendo a la clasificación funcional \\ Physical fitness analysis according the functional classification in national wheel- chair basketball players
}

\author{
Javier Yanci $^{1}$, Aitor Iturricastilloํ, Lander Lozano ${ }^{2}$, Cristina Granados ${ }^{1}$
}

1.Facultad de Ciencias de la Actividad Física y del Deporte, Universidad del País Vasco, UPV/EHU, Vitoria-Gasteiz, España.

2.Club Deportivo Zuzenak, Vitoria-Gasteiz, España.

\section{Resumen}

Los objetivos de este estudio fueron, por un lado, analizar la condición física de jugadores de baloncesto en silla de ruedas, y por otro, analizar las diferencias existentes entre estos deportistas con distintos niveles de clasificación funcional. En este estudio participaron 15 jugadores masculinos de baloncesto en silla de ruedas $(38,73 \pm 4,49$ años, 78,49 $\pm 10,89 \mathrm{~kg}, 89,96 \pm 3,58 \mathrm{~cm}$ talla sentados), que fueron divididos en dos grupos en función de la clasificación funcional: Categoría $A$ ( $n=7$, jugadores con un alto nivel de afectación funcional) y Categoría $B(n=8$, jugadores con un menor nivel de afectación). Los resultados obtenidos determinan que el grupo con mayor clasificación funcional obtuvo mejores resultados en el test Yo-Yo $10 \mathrm{~m}$ (distancia recorrida, 19,32\%, d = 0,89, p > 0,05). Sin embargo, las diferencias entre grupos únicamente se observaron en los test específicos de aceleración $5 \mathrm{~m}$ con balón $(p>0,05,6,84 \%$, $d=0,86), 20 \mathrm{~m}$ con balón $(p<0,01,7,59 \%, d=2,23)$ y Pick up $(p<0,05,10,07 \%, d=0,87)$ y en la fuerza medida a través del test de lanzamiento de balón medicinal ( $p<0,05,13,82 \%, d=0,73$ ). Sin embargo, estas diferencias entre grupos no se observaron en la capacidad de aceleración sin balón, en el T-test o en los test de fuerza handgrip o maximal pass. La clasificación funcional puede afectar a la condición física aeróbica, a la fuerza explosiva medida a través del test de lanzamiento de balón medicinal, así como a los test específicos de aceleración de 5 y $20 \mathrm{~m}$ con balón.

Palabras clave: valoración física; fuerza, agilidad; baloncesto en silla de ruedas.

\section{Abstract}

The objectives of this study were, first, to analyze the physical fitness in wheelchair basketball players, and secondly, to analyze the differences between athletes with different levels of functional classification. The study included 15 male wheelchair basketball players $(38.73 \pm 4.49$ years, $78.49 \pm 10.89 \mathrm{~kg}, 89.96$ $\pm 3.58 \mathrm{~cm}$ sitting height), which were divided into two groups according on the impairment: Category A ( $n=7$, players with a high level of functional impairment) and Category $B(n=8$ players with a lower level of functional impairment). The results determined that the group with greater functional classification performed better Yo-Yo $10 \mathrm{~m}$ test (distance covered, $19.32 \%, d=0.89, p>0.05$ ). However, the differences between groups are observed in the specific acceleration $5 \mathrm{~m}$ with ball test $(\mathrm{p}>0.05,6.84 \%$, $d=0.86), 20 \mathrm{~m}$ with ball $(\mathrm{p}<0.01,7.59 \%, d=2.2)$ and Pick up $(\mathrm{p}<0.05,10.07 \%, d=0.87)$ and the strength measured by the medicine ball test $(p<0,05,13.82 \%, d=0,73)$. However, these differences between groups were not observed in the acceleration capacity without the ball, the T-test, maximal pass or handgrip test. The functional classification can affect aerobic fitness, the explosive force, as well as specific test with ball.

Key words: physical fitness; heart rate; strength; wheelchair basketball. 
Yanci, J.; Iturricastillo, A.; Lozano, L.; Granados, C. (2015). Análisis de la condición física de jugadores nacionales de baloncesto en silla atendiendo a la clasificación funcional. RICYDE. Revista internacional de ciencias del deporte, 40(11), 173-185. http://dx.doi.org/10.5232/ricyde2015.04006

\section{Introducción}

$\mathrm{M}$ uchos estudios científicos demuestran los beneficios de la práctica regular de actividad física para la población general (Bouchard, Shephard, Stephens, Sutton, y McPherson, 1990; Shephard, 1995) y también para personas usuarias de silla de ruedas (Bhambhani, 2002). Estos beneficios no sólo se ven reflejados en el ámbito físico, sino también son de gran valor en el plano psicológico, por lo que una adecuada practica de actividad física puede ser una actividad fundamental para la rehabilitación de las personas con gran discapacidad (Sherrill, 2004; Van der Woude, De Groot, y Janssen, 2006). La investigación actual, en distintas áreas de conocimiento, ha centrado su interés por los efectos físicos, psicológicos y sociales de la actividad deportiva en personas con discapacidad física (Banack, Sabiston, y Bloom, 2011; Hutzler, Chacham-Guber, y Reiter, 2013). Algunos estudios muestran que la participación en deporte es un componente importante para la calidad de vida percibida y para la integración social de las personas con discapacidad (Bhambhani, 2002; McVeigh, Hitzig, y Craven, 2009).

En este sentido, la participación en programas específicos de actividad física y de práctica deportiva han impulsado firmemente a las personas con discapacidad para el desarrollo de sus habilidades psicosociales, el rendimiento y la competencia deportiva (Hellison, 2003; Sherrill, 2004). Sin embargo, la práctica de actividad física en el tiempo de ocio en personas con discapacidad es especialmente más reducida que la de las personas sin discapacidad (Longmuir y Bar-Or, 2000; Rimmer, 2001). La baja tasa de participación está ligada a las barreras percibidas, así como a la reducción de las oportunidades para la actividad deportiva en un grupo (Jaarsma, Dijkstra, Geertzen, y Dekker, 2014; Kolt, Kirkby, Bar-Eli, Blumenstein, Chadha, y Liu, 1999). Por lo tanto, uno de los retos de los profesionales de la actividad física y del deporte, así como de los profesionales sanitarios y rehabilitadores puede ser el promover la práctica de ejercicio físico entre las personas con algún tipo de discapacidad.

Varias investigaciones han determinado que la condición física es un buen predictor de morbilidad y mortalidad (Erikssen, 2001; Myers, Kaykha, George, Abella, Zaheer, Lear, Yamazaki, y Froelicher, 2004). En este sentido, las directrices de actividad física están destinadas a mejorar la condición física general de las personas, no sólo para reducir la morbilidad y mortalidad asociada al sedentarismo, sino también para mejorar la calidad de vida (Peterson y col., 2009). Una investigación anterior ha determinado que las funciones psicomotoras y la condición física se ven afectados en las personas usuarias de silla de ruedas después de una lesión de la médula espinal, amputaciones o infecciones del sistema nervioso como la poliomielitis (Plinta, Saulicz, Gnat, Juras, y Waskiewicz, 2005). De la misma forma, algunos estudios realizados con deportistas con discapacidad exponen que la clasificación funcional puede condicionar el nivel de aptitud física en distintos test de aceleración, manejo de la silla o fuerza muscular (Molik, Laskin, Kosmol, Marszalek, Morgule-Adamowicz, y Frick, 2013). Varios estudios realizados con deportistas con discapacidad y con jugadores de baloncesto en silla de ruedas (BSR) han centrado sus esfuerzos en determinar si existen diferencias en la condición física entre deportistas con altas afectaciones y deportistas con afectaciones más leves (De Lira y col., 2010; Vanlandewijck y col., 2004). Sin embargo, debido a los resultados contradictorios obtenidos en los distintos estudios (De Lira y col., 2010; Molik, Kosmol, Morgulec, Hübner-Woźniak, y Rutkowska, 2006; Molik, Laskin, Kosmol, Skucas, y Bida, 2010; Vanlandewijck y col., 2004), son necesarios más investigaciones al respecto para determinar si el nivel de clasificación funcional influye en la condición física de deportistas con discapacidad física y concretamente en jugadores de baloncesto en silla de ruedas. 
Yanci, J.; Iturricastillo, A.; Lozano, L.; Granados, C. (2015). Análisis de la condición física de jugadores nacionales de baloncesto en silla atendiendo a la clasificación funcional. RICYDE. Revista internacional de ciencias del deporte, 40(11), 173-185. http://dx.doi.org/10.5232/ricyde2015.04006

En el ámbito deportivo, los test de laboratorio han sido ampliamente utilizados para evaluar el rendimiento físico y físiológico de jugadores de baloncesto en silla de ruedas (GooseyTolfrey, 2005, Leicht, Bishop, y Goosey-Tolfrey, 2012). Sin embargo, a pesar de que la validez y fiabilidad de estos test de laboratorio ha sido ampliamente probada, los test de campo también han sido muy utilizados para medir la condición física en esta modalidad deportiva (De Groot, Balvers, Kouwenhoven, y Janssen, 2012, Molik y col., 2013, Vanlandewijck, Daly, y Theisen, 1999). La utilización de los test de campo puede ser más apropiada ya que presentan una mayor validez ecológica. Estos test permiten evaluar de forma simultánea a un gran número de deportistas, son generalmente más baratos, más fáciles y rápidos de aplicar. Varios estudios han analizado la capacidad de aceleración, la agilidad, la fuerza muscular y el rendimiento aeróbico (De Groot et al., 2012, Goosey-Tolfrey y Tolfrey, 2008, Vanlandewijck y col., 1999) de jugadores de BSR. Sin embargo, la mayor parte de estos estudios están realizados con jugadores de alto nivel internacional y no existe un consenso en cuanto a las diferencias atendiendo a la clasificación funcional (Molik et al., 2013, Molik y col., 2010).

Los objetivos de este estudio fueron, por un lado, analizar la condición física (capacidad aeróbica, capacidad de aceleración, cambio de dirección y fuerza muscular) de jugadores de categoría nacional de baloncesto en silla de ruedas, y por otro, analizar las diferencias existentes entre estos deportistas con distintos niveles de clasificación funcional.

\section{Método}

\section{Participantes}

Quince jugadores masculinos de BSR $(38,73 \pm 4,49$ años, 78,49 $\pm 10,89 \mathrm{~kg}, 89,96 \pm 3,58 \mathrm{~cm}$ talla sentados), pertenecientes a un equipo que militaba en la primera división de la Liga española de BSR participaron en este estudio. Los participantes fueron divididos en dos grupos (Molik y col., 2013) en función de la clase funcional (IWBF, 2010). Categoría A (n = $7 ; 41,86 \pm 5,08$ años; 77,00 $\pm 14,65 \mathrm{~kg} ; 89,96 \pm 5,12 \mathrm{~cm}$ talla sentados): jugadores clasificados en una clase funcional de la International Wheelchair Basketball Federation (IWBF) igual o inferior a 2,5 puntos. Categoría $\mathrm{B}(\mathrm{n}=8 ; 36,00 \pm 1,69$ años; 79,97 $\pm 7,54 \mathrm{~kg}$; $89,96 \pm 2,24 \mathrm{~cm}$ talla sentados): jugadores clasificados en una clase funcional IWBF superior a 2,5 puntos. Todos los participantes entrenaban dos sesiones semanales con una duración por sesión de $150 \mathrm{~min}$ y disputaban un partido oficial cada semana durante el periodo competitivo. A todos los participantes se les explicaron los riesgos y beneficios de la participación en el estudio, firmaron el preceptivo consentimiento informado y podían retirarse del mismo en cualquier momento. El estudio fue aprobado por el Comité de Ética de la Universidad del País Vasco, UPV/EHU.

\section{Procedimiento}

Las pruebas se realizaron en la cancha de baloncesto donde entrenaba el equipo, siempre entre las 19:00 y 21:00 horas, una semana antes del inicio de la temporada oficial. Todos los jugadores conocían la ejecución correcta de los test ya que eran las pruebas habituales de valoración de la condición física realizados por el equipo en las temporadas anteriores. Los jugadores fueron instruidos para realizar todas las pruebas a la máxima intensidad. Las pruebas se realizaron en dos sesiones diferentes con dos días de diferencia. Durante la primera sesión, cada participante realizó los test de aceleración con y sin balón, y las pruebas de agilidad. En la segunda sesión, se realizaron los test de fuerza y el test de resistencia. Antes de cada sesión de test se realizó un calentamiento estándar que consistía en 5 min de desplazamiento a baja intensidad con la silla de ruedas, dos aceleraciones de $15 \mathrm{~m}$ y 3 
Yanci, J.; Iturricastillo, A.; Lozano, L.; Granados, C. (2015). Análisis de la condición física de jugadores nacionales de baloncesto en silla atendiendo a la clasificación funcional. RICYDE. Revista internacional de ciencias del deporte, 40(11), 173-185. http://dx.doi.org/10.5232/ricyde2015.04006

lanzamientos máximos de balón. Todos los participantes realizaron las pruebas con su silla deportiva habitual.

Test de aceleración con y sin balón: Los participantes realizaron tres aceleraciones máximas de $20 \mathrm{~m}$ sin balón y otras tres con balón (Vanlandewijck y col., 1999), con un descanso de 120 s entre cada repetición (Abt, Siegler, Akubat, y Castagna, 2011). Los participantes salían desde una posición de $0,5 \mathrm{~m}$ con respecto a la primera fotocélula y comenzaban cuando ellos lo consideraban oportuno (Castagna, Impellizzeri, Chaouachi, Ben Abdelkrim, y Manzi, 2011). El tiempo empleado en cada repetición se registró utilizando tres fotocélulas (Microgate Polifemo Radio Ligth ${ }^{\circledR}$, Bolzano, Italia) colocadas a una altura de $0,4 \mathrm{~m}$ (Gorostiaga, Izquierdo, Ruesta, Iribarren, González-Badillo, e Ibañez, 2004). El temporizador se activaba automáticamente cuando los participantes pasaban por la primera fotocélula y se registró el tiempo intermedio a los $5 \mathrm{~m}$ (De Groot y col., 2012) y el tiempo final a los $20 \mathrm{~m}$ (Vanlandewijck y col., 1999). En los esprines con balón se debía cumplir el reglamento marcado por la IWBF tal y como había descrito anteriormente De Groot y col. (2012). El coeficiente de variación (CV) para estos test entre las distintas repeticiones realizadas fue inferior a $2,91 \%$.

Test de agilidad T-test: Los participantes debían completar el circuito marcado en la figura 1 atendiendo al protocolo utilizando anteriormente por Sassi, Dardouri, Yahmed, Gmada, Mahfoudhi, y Gharbi (2009), con la modificación de que los desplazamientos se realizaban de forma libre y se tocaba la parte superior de los conos (Yanci, Reina, Los Arcos, y Cámara, 2013; Yanci, Granados, Otero, Badiola, Olasagasti, Bidaurrazaga, Iturricastillo, y Gil, 2015). Todos los participantes realizaron la prueba 3 veces con un mínimo de 3 min de descanso entre las repeticiones. La altura de los conos fue de $0.3 \mathrm{~m}$. Se utilizó una fotocélula (Microgate Polifemo Radio Ligth ${ }^{\circledR}$, Bolzano, Italia) situado sobre el cono A para registrar el tiempo. El CV para este test entre las distintas repeticiones realizadas fue de $3,45 \%$.

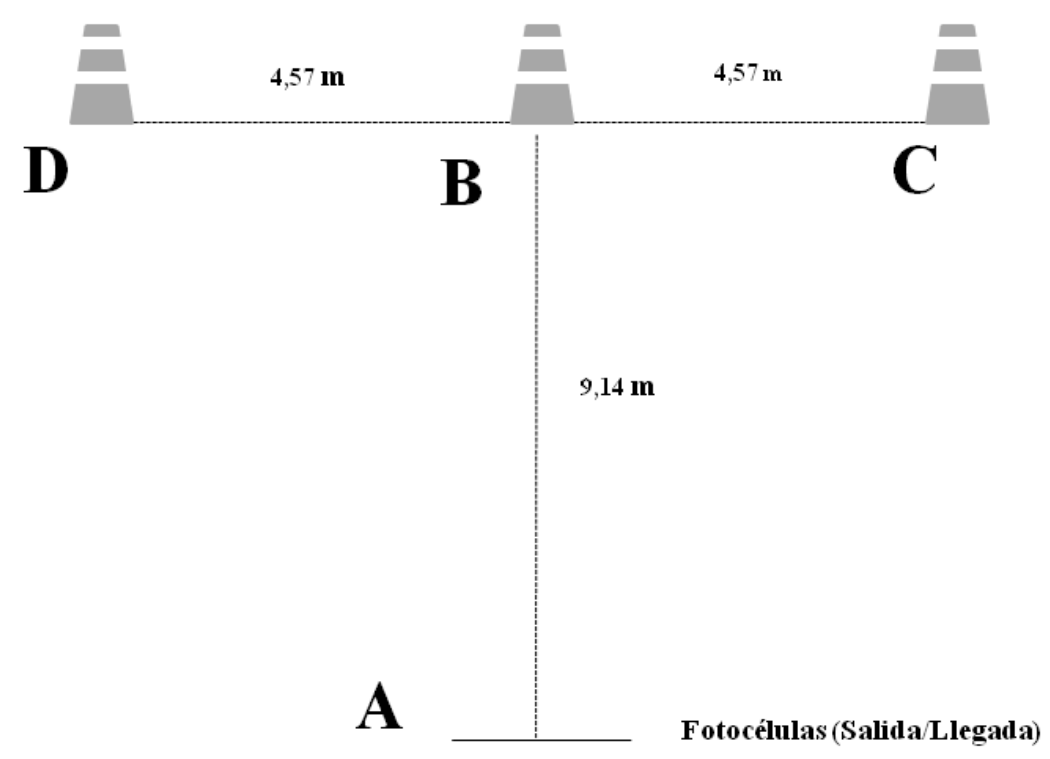

Figura 1. Recorrido para el test de agilidad T-Test.

Test de agilidad Pick up: Atendiendo al protocolo establecido por De Groot y col. (2012) y partiendo desde una posición de parado, el deportista debía comenzar a impulsar la silla e ir recogiendo cuatro balones de baloncesto situados en el suelo (Figura 2), dos veces con la mano izquierda y dos veces con la mano derecha. Después de recoger la pelota, ésta debía ser 
Yanci, J.; Iturricastillo, A.; Lozano, L.; Granados, C. (2015). Análisis de la condición física de jugadores nacionales de baloncesto en silla atendiendo a la clasificación funcional. RICYDE. Revista internacional de ciencias del deporte, 40(11), 173-185. http://dx.doi.org/10.5232/ricyde2015.04006

colocada en el regazo y había que empujar la silla por lo menos dos veces antes de desprenderse del balón (De Groot y col., 2012). El tiempo total para completar la prueba fue registrado mediante dos fotocélulas (Microgate Polifemo Radio Ligth ${ }^{\circledR}$, Bolzano, Italia) situadas al inicio y al final del recorrido. Todos los participantes realizaron la prueba 3 veces, con un mínimo de 3 min de descanso. El CV para este test entre las distintas repeticiones realizadas fue de $3,83 \%$.

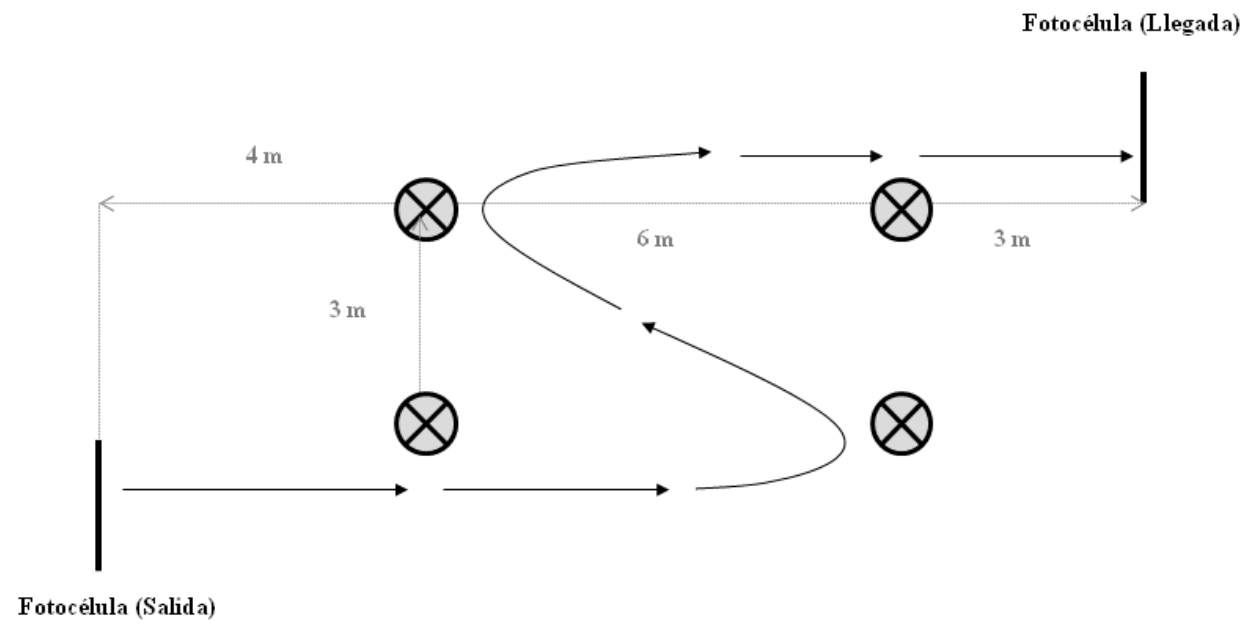

Figura 2. Recorrido para el test de agilidad Pick up.

Test handgrip: La fuerza del antebrazo se midió en la mano dominante (Molik y col., 2013; Yanci y col., 2015) mediante un dinamómetro hidráulico de mano portátil (5030J1, Jamar ${ }^{\mathbb{B}}$, Sammons Preston, Inc, Reino Unido). La prueba se realizó en la posición sentada en la silla de ruedas y con el brazo totalmente extendido. El protocolo de prueba consistió en tres contracciones isométricas máximas de $5 \mathrm{~s}$, con un período de descanso de al menos $60 \mathrm{~s}$. El CV para este test entre las distintas repeticiones realizadas fue de 1,87\%.

Maximal pass: Los participantes situados en la línea marcada realizaban 5 lanzamientos bilaterales de un balón de baloncesto lo más lejos posible (De Groot y col., 2012). Se midió la distancia $(\mathrm{m})$ entre la línea marcada y el lugar donde el balón dio el primer bote. La puntuación final para el análisis estadístico fue la distancia media de los cinco lanzamientos (De Groot y col., 2012). El CV para este test entre las distintas repeticiones realizadas fue de $3,64 \%$.

Test lanzamiento de balón medicinal: Colocados en una posición idéntica al test de maximal pass, los participantes tenían que lanzar un balón medicinal de $5 \mathrm{~kg}$ lo más lejos posible (Gonaus y Muller, 2012). Cada participante realizó tres intentos y se midió la distancia (m) desde la línea de lanzamiento hasta el lugar donde el balón realizaba el primer contacto con el suelo. El CV para este test entre las distintas repeticiones realizadas fue de 3,12\%.

Test resistencia Yo-Yo intermitente recovery $10 \mathrm{~m}$ : Se utilizó la versión 1 del test Yo-Yo (YYIR1 $10 \mathrm{~m}$ ) previamente descrito por Yanci y col. (2015) para jugadores de BSR. Los jugadores debían de recorrer una distancia de $10 \mathrm{~m}$ de ida y otros $10 \mathrm{~m}$ de vuelta en lugar de los $20 \mathrm{~m}$ del test original (Castagna, Impellizzeri, Rampinini, D'Ottavio, y Manzi, 2008). La velocidad de carrera se marcaba mediante un sistema de audio previamente programado. La finalización del test para cada jugador se consideró cuando no llegaban a la línea correspondiente en el tiempo marcado por segunda vez (evaluación objetiva) o cuando el propio participante consideraba que no podía continuar en la prueba (evaluación subjetiva), midiéndose la distancia total recorrida por cada jugador (Castagna y col., 2008) y la 
Yanci, J.; Iturricastillo, A.; Lozano, L.; Granados, C. (2015). Análisis de la condición física de jugadores nacionales de baloncesto en silla atendiendo a la clasificación funcional. RICYDE. Revista internacional de ciencias del deporte, 40(11), 173-185. http://dx.doi.org/10.5232/ricyde2015.04006

percepción subjetiva del esfuerzo (RPE) al final de la prueba (Foster, Florhaug, Franklin, Gottschall, Hrovatin, Parker, Doleshal, y Dodge, 2001). La frecuencia cardíaca (FC) fue monitorizada durante toda la prueba cada $5 \mathrm{~s}$ (Polar Team System Sport ${ }^{\circledR}$, Polar Electro Oy, Finlandia). Justo al finalizar la prueba se obtuvo una muestra de sangre del lóbulo de la oreja (Lactate Pro LT-1710 ${ }^{\circledR}$, Arkray Inc Ltd, Kyoto, Japón) con el fin de analizar la concentración de lactato [LA]. Posteriormente se recogieron muestras salivares (Granger, Schwartz, Booth, y Arentz, 1999) en frascos de plástico individuales de $20 \mathrm{ml}$ con el fín de analizar las concentraciones de cortisol $\left(\mathrm{g} \cdot \mathrm{dl}^{-1}\right)$ y testosterona $\left(\mathrm{pg} \cdot \mathrm{ml}^{-1}\right)$. Así mismo, se solicitó a todos los jugadores la percepción subjetiva del esfuerzo a nivel respiratorio (RPEres) y muscular (RPEmus) atendiendo a las consideraciones realizadas por Los Arcos, Yanci, Mendiguchia, y Gorostiaga (2014).

\section{Análisis estadístico de los datos}

Los resultados se presentan como media \pm desviación típica (DT) de la media. La normalidad de los datos se analizó mediante las pruebas de Kolmogorov-Smirnov y Saphiro-Wilks, con el fin de verificar la necesidad de pruebas paramétricas o no paramétricas. Se optó por realizar pruebas no paramétricas en el caso de la comparación entre grupos dado que la muestra por grupo fue menor de 10 participantes. Para realizar el análisis estadístico se utilizó el mejor registro de cada tipo de prueba, salvo en el test Maximal pass, donde se utilizó el promedio de todos los lanzamientos (De Groot y col., 2012). Para determinar las diferencias existentes entre los resultados de los dos grupos (Categoría A y B) en las distintas variables analizadas (aceleración con y sin balón, agilidad, fuerza y resistencia) se utilizó la prueba U de MannWhitney. El tamaño del efecto (TE) se calculó atendiendo al método propuesto por Cohen (1988). Tamaños del efecto menores a 0,2 , entre $0,2-0,5$, entre $0,5-0,8$ o mayores de 0,8 fueron considerados trivial, bajo, moderado o alto, respectivamente. El análisis estadístico se realizó con el programa Statistical Package for Social Sciences (SPSS Inc, versión 20,0 Chicago, IL, EE.UU.). El nivel de significación estadística fue establecido en $p<0,05$.

\section{Resultados}

Los resultados en la capacidad de aceleración en 5 y $20 \mathrm{~m}$ sin balón y 5 y $20 \mathrm{~m}$ con balón para todo el grupo fueron de $1,72 \pm 0,08 \mathrm{~s}, 5,16 \pm 0,18 \mathrm{~s}, 1,82 \pm 0,17 \mathrm{~s}$ y $5,68 \pm 0,37 \mathrm{~s}$, respectivamente. Los jugadores de la categoría A obtuvieron peores resultados en el test de 20 $\mathrm{m}$ con balón $(\mathrm{p}<0,01$, diferencias de medias $=7,59 \%, \mathrm{~d}=2,23)$ y en el de $5 \mathrm{~m}$ con balón $(\mathrm{p}>$ 0.05 , diferencia de medias $=6,84 \%, \mathrm{~d}=0,86)$ en comparación con los jugadores de la categoría B (Figura 3). Sin embargo, no se encontraron diferencias significativas entre las categorías y los tamaños del efecto fueron moderados en la capacidad de aceleración sin balón tanto en $5 \mathrm{~m}(\mathrm{p}>0.05$, diferencia de medias, 3,00\%, $\mathrm{d}=0,66)$ como en $20 \mathrm{~m}(\mathrm{p}>0.05$, diferencia de medias $=-2,11 \%, d=0,58)$. 
Yanci, J.; Iturricastillo, A.; Lozano, L.; Granados, C. (2015). Análisis de la condición física de jugadores nacionales de baloncesto en silla atendiendo a la clasificación funcional. RICYDE. Revista internacional de ciencias del deporte, 40(11), 173-185. http://dx.doi.org/10.5232/ricyde2015.04006

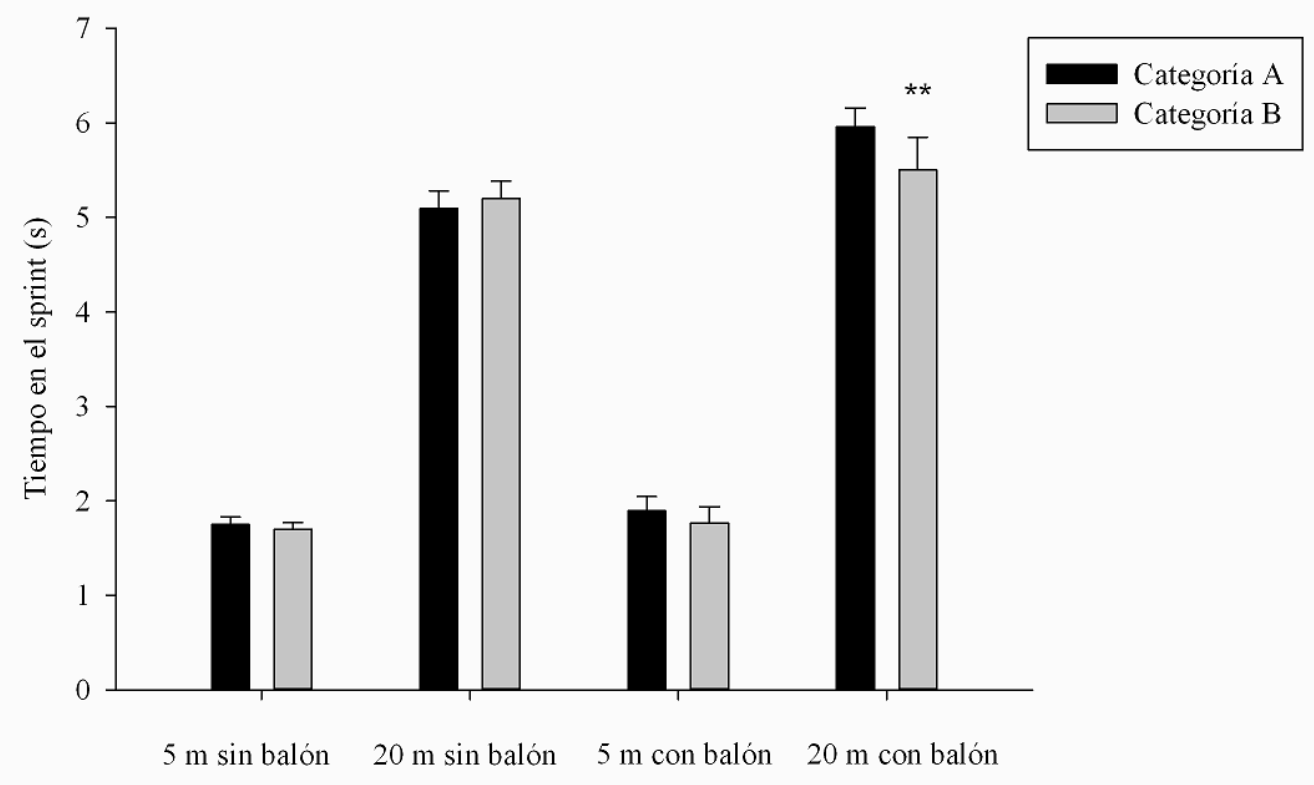

Figura 3. Resultados en la capacidad de aceleración con y sin balón para las categorías A y B.

** Diferencias significativas $(\mathrm{p}<0,01)$ entre la categoría A y B.

Los resultados para todo el grupo en el T-test y en el Pick up fueron de 14,36 $\pm 0,70 \mathrm{~s}$ y 12,00 $\pm 1,14 \mathrm{~s}$, respectivamente. Se encontraron diferencias significativas $(\mathrm{p}<0,05$, diferencia de medias $=10,07 \%, d=0,87$ ) entre la categoría A y la B en el test de Pick up (Figura 4). Sin embargo, estas diferencias no se observaron en el T-test $(\mathrm{p}>0,05$, diferencia de medias $=$ $2,56 \%)$ y el tamaño del efecto fue moderado $(d=0,55)$.

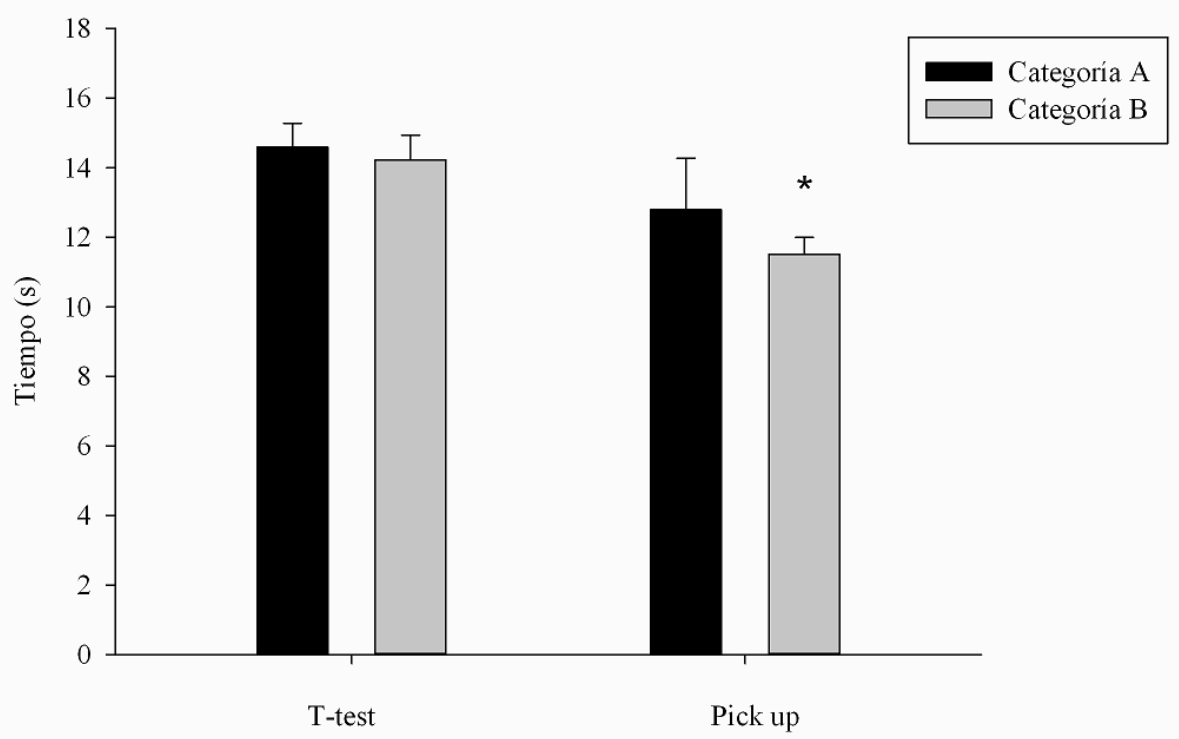

Figura 4. Resultados en función de la categoría (A y B) en los test de agilidad.

*Diferencias significativas $(\mathrm{p}<0,05)$ entre categoría A y B. 
Yanci, J.; Iturricastillo, A.; Lozano, L.; Granados, C. (2015). Análisis de la condición física de jugadores nacionales de baloncesto en silla atendiendo a la clasificación funcional. RICYDE. Revista internacional de ciencias del deporte, 40(11), 173-185. http://dx.doi.org/10.5232/ricyde2015.04006

Los resultados en los test de fuerza y resistencia se muestran en la tabla 1. Se encontraron diferencias significativas entre categorías en el test de lanzamiento de balón medicinal y en los valores declarados de RPEres al finalizar la prueba de resistencia. Los jugadores de la categoría A recorrieron menos metros $(\mathrm{p}>0,05, \mathrm{~d}=0,89)$ en el test YYIR1 $10 \mathrm{~m}$ que los jugadores de la categoría B. Los valores de cortisol y testosterona al final de la prueba de resistencia fueron diferentes entre las categorías $(p>0,05, \mathrm{~d}=0,73 \mathrm{y} d=1,15$ respectivamente).

Tabla 1. Resultados en los test de fuerza y resistencia para el total de jugadores y los jugadores de la categoría A y B.

\begin{tabular}{|c|c|c|c|c|c|}
\hline Variable & Total & Categoría $\mathrm{A}$ & Categoría B & Dif. (\%) & $\mathrm{d}$ \\
\hline \multicolumn{6}{|l|}{ Fuerza } \\
\hline Handgrip (kg) & $58,20 \pm 9,56$ & $58,57 \pm 12,73$ & $57,88 \pm 6,60$ & 1,19 & 0,05 \\
\hline Maximal pass (m) & $12,92 \pm 2,33$ & $12,47 \pm 3,08$ & $13,31 \pm 1,54$ & $-6,69$ & 0,27 \\
\hline Balón medicinal (m) & $4,87 \pm 0,71$ & $4,54 \pm 0,86$ & $5,16 \pm 0,40^{*}$ & $-13,82$ & 0,73 \\
\hline \multicolumn{6}{|l|}{ Resistencia } \\
\hline Lactato $\left(\mathrm{mmol} \cdot \mathrm{l}^{-1}\right)$ & $8,67 \pm 3,78$ & $8,81 \pm 4,96$ & $8,55 \pm 2,73$ & 3,00 & 0,05 \\
\hline FCmax (latidos $\left.\cdot \min ^{-1}\right)$ & $182,14 \pm 13,75$ & $177,71 \pm 18,38$ & $186,57 \pm 5,09$ & $-4,98$ & 0,48 \\
\hline Distancia (m) & $1.366,67 \pm 280,07$ & $1.257,14 \pm 274,09$ & $1.500,00 \pm 247,04$ & $-19,32$ & 0,89 \\
\hline RPEres & $5,75 \pm 2,69$ & $4,07 \pm 2,37$ & $7,43 \pm 1,88 * *$ & $-82,46$ & 1,42 \\
\hline RPEmus & $5,64 \pm 2,16$ & $6,07 \pm 2,39$ & $5,21 \pm 2,00$ & 14,12 & 0,36 \\
\hline Cortisol $\left(\mu \mathrm{g} \cdot \mathrm{dl}^{-1}\right)$ & $0,30 \pm 0,32$ & $0,46 \pm 0,41$ & $0,16 \pm 0,09$ & 65,97 & 0,73 \\
\hline Testosterona $\left(\mathrm{pg} \cdot \mathrm{ml}^{-1}\right)$ & $138,47 \pm 62,50$ & $120,00 \pm 30,18$ & $154,63 \pm 79,95$ & $-28,85$ & 1,15 \\
\hline
\end{tabular}

Dif. = diferencia de medias, $\mathrm{d}=$ tamaño del efecto, $\mathrm{RPEres}=$ percepción subjetiva del esfuerzo respiratoria, RPEmus $=$ percepción subjetiva del esfuerzo muscular. Diferencias significativas entre categoría A y B, * p $<0,05, * * \mathrm{p}<0,01$.

\section{Discusión}

El análisis de la condición física en jugadores de BSR con algún tipo de discapacidad física puede aportar una información relevante para determinar su rendimiento deportivo. El principal objetivo de este estudio fue analizar la condición física en deportistas de baloncesto en silla de ruedas. De la misma forma, se analizaron las diferencias existentes entre deportistas con distintos niveles de clasificación funcional. Los resultados obtenidos determinan que el grupo con menor afectación funcional obtuvo mejores resultados en el rendimiento aeróbico. Sin embargo, las diferencia entre grupos para la capacidad anaeróbica únicamente se observó en los test específicos con balón (aceleración 5 y $20 \mathrm{~m}$ con balón y Pick up) y en la fuerza medida a través del test de lanzamiento de balón medicinal. Sin embargo, estas diferencias entre grupos no se observaron en la capacidad de aceleración sin balón, en el T-test o en los test de fuerza handgrip o maximal pass.

Estudios anteriores han determinado que un mejor manejo de la silla de ruedas puede ayudar a obtener un mayor rendimiento deportivo en competición (De Groot y col., 2013) además de mejorar la calidad de vida (Nash, Van de Ven, Van Elk, y Johnson, 2007). Los resultados obtenidos en este estudio en el T-test, son mejores que los obtenidos por Yanci y col. (2015) en jugadores de la tercera división española. De la misma forma, los resultados obtenidos en la capacidad de aceleración y en el test de agilidad Pick up también fueron mejores que los 
Yanci, J.; Iturricastillo, A.; Lozano, L.; Granados, C. (2015). Análisis de la condición física de jugadores nacionales de baloncesto en silla atendiendo a la clasificación funcional. RICYDE. Revista internacional de ciencias del deporte, 40(11), 173-185. http://dx.doi.org/10.5232/ricyde2015.04006

reportados por De Groot y col. (2012). Posiblemente el nivel de los participantes y las diferentes metodologías de medición de los test han podido influir en los resultados obtenidos. En el presente estudio no se obtuvieron diferencias significativas atendiendo a la clasificación funcional en los test de aceleración 5 y $20 \mathrm{~m}$ ni en el T-test. Sin embargo, si se obtuvieron diferencias en los test anaeróbicos con balón (aceleración 5 y 20 m con balón y Pick up). Atendiendo a estos resultados, la condición física en las variables de capacidad de aceleración y agilidad no se ve influenciada por la clasificación funcional, sin embargo, los jugadores con menor afectación funcional obtienen mejores resultados en los test específicos con balón. Estos datos contrastan con los obtenidos por Molik y col. (2013) ya que estos autores describen diferencias significativas en función de la clasificación en jugadoras de baloncesto en silla de ruedas tanto en la capacidad de aceleración, como en los test de agilidad slalom con y sin balón. Estos resultados contradictorios pueden deberse a los distintos test utilizados, así como a la diferencia de género de los participantes.

Los resultados obtenidos en este estudio son mejores que los obtenidos por Yanci y col. (2015) en jugadores de BSR de una categoría deportiva más baja (tercera división española) en cuanto a los test de handgrip (58,20 vs. $44,50 \mathrm{~kg})$ y maximal pass $(12,92 \mathrm{vs} .8,39 \mathrm{~m})$. Sin embargo, De Groot y col. (2012) obtuvieron resultados superiores (12,92 vs. 13,3 m) en el test maximal pass. Son necesarios más estudios que determinen si el nivel competitivo influye en el rendimiento de estas dos variables analizadas. A pesar de que en los test de handgrip y maximal pass no se obtuvieron diferencias entre categorías (A y B), la prueba de lanzamiento de balón medicinal arrojó diferencias entre los dos grupos. Los jugadores con mayor clasificación funcional obtuvieron mejores resultados en este test de fuerza explosiva. El BSR incluye una gran variedad de manifestaciones de la fuerza muscular del miembro superior en acciones específicas del juego como acelerar, frenar, cambiar de dirección con la silla, lanzar, driblar o rebotear (Wang, Chen, Limroongreungrat, y Change, 2005). Estas acciones específicas requieren altos niveles de fuerza muscular de los brazos y del tronco. En nuestro estudio no se encontraron diferencias en los test de handgrip y maximal, donde no se utilizaban implementos pesados. Sin embargo estas diferencias sí se manifestaron en el test de lanzamiento de balón medicinal. Posiblemente este test presente una mayor implicación de la acción del tronco y este aspecto ha podido influir en las diferencias encontradas entre los distintos grupos. Debido a la disparidad de resultados obtenidos en función de la clasificación funcional en los test de fuerza de nuestro estudio, son necesarios más estudios al respecto así como analizar si puede resultar beneficioso implementar programas específicos de fuerza en las extremidades superiores en jugadores de BSR con altas afectaciones.

Los jugadores con mayor clasificación funcional de este estudio obtuvieron mejores resultados en el test de resistencia, concretamente en la distancia total recorrida $(\mathrm{p}>0,05$, $19 \%, d=0,89)$. Este aspecto nos lleva a pensar que los deportistas con menor clasificación funcional tienen un menor rendimiento aeróbico. Así mismo, los deportistas más afectados obtuvieron mayores niveles de cortisol $(p>0,05, d=0,73)$ y menores niveles de testosterona $(\mathrm{p}>0,05, \mathrm{~d}=1,15)$ al final de la prueba de resistencia. La resistencia aeróbica ha sido definida como uno de los componentes fundamentales del rendimiento en competición en deportes de silla de ruedas (Coutts, 1998; Croft, Dybrus, Lenton, y Goosey-Tolfrey, 2010). Estos autores determinan que la implicación de la capacidad aeróbica es importante concretamente en BSR. Por lo tanto, sería interesante poder implementar programas específicos para la mejora de la capacidad aeróbica en deportistas con discapacidad física con altas afectaciones con el fin de que estos jugadores puedan afrontar el juego y la competición con mayor garantía. Sin embargo, debido a la disparidad de test utilizados por diferentes autores, la comparación de resultados resulta complicada. 
Yanci, J.; Iturricastillo, A.; Lozano, L.; Granados, C. (2015). Análisis de la condición física de jugadores nacionales de baloncesto en silla atendiendo a la clasificación funcional. RICYDE. Revista internacional de ciencias del deporte, 40(11), 173-185. http://dx.doi.org/10.5232/ricyde2015.04006

\section{Conclusiones}

Los resultados obtenidos en este estudio ponen de manifiesto que el grupo con mayor clasificación funcional obtuvo mejores resultados en la capacidad aeróbica. Sin embargo, las diferencias entre grupos para la capacidad anaeróbica únicamente se observaron en los test específicos con balón y en la fuerza medida a través del test de lanzamiento de balón medicinal. Por el contrario, estas diferencias entre grupos no se observaron en la capacidad de aceleración sin balón, en el T-test o en los test de fuerza handgrip o maximal pass. De esta forma, los jugadores con mayor clasificación funcional han mostrado mejores valores en los test específicos con balón. Sin embargo estos resultados no han sido similares en los test sin balón. Son necesarios más estudios donde se analicen distintas variables de condición física en deportistas de distintos niveles de clasificación funcional.

\section{Agradecimientos}

Queremos agradecer al C.D. Zuzenak por ofrecernos la posibilidad de realizar este estudio de investigación y en concreto al entrenador y a los jugadores participantes en el mismo.

\section{Referencias}

Abt, G.; Siegler, J. C.; Akubat, I., \& Castagna, C. (2011). The effects of a constant sprint-to-rest ratio and recovery mode on repeated sprint performance. Journal of Strength and Conditioning Research, 25(6), 1695-1702.

http://dx.doi.org/10.1519/JSC.0b013e3181dbdc06

Banack, H. R.; Sabiston, C. M., \& Bloom, G. A. (2011). Coach autonomy support, basic need satisfaction, and intrinsic motivation of paralympic athletes. Research Quarterly in Exercise and Sport, 82(4), 722-730.

http://dx.doi.org/10.1080/02701367.2011.10599809

Bhambhani, Y. (2002). Physiology of wheelchair racing in athletes with spinal cord injury. Sports Medicine, 32(1), 23-51.

http://dx.doi.org/10.2165/00007256-200232010-00002

Bouchard, C.; Shepard, R. J.; Stephens, T.; Sutton, J. R., \& McPherson, B. D. (eds). (1990). Exercise, fitness and health: A consensus of current knowledge. Champaign, Ilinois: Human Kinetics.

Castagna, C.; Impellizzeri, F. M.; Rampinini, E.; D'Ottavio, S., \& Manzi V. (2008). The Yo-Yo intermittent recovery test in basketball players. Journal of Science and Medicine in Sport, 11(2), 202-208.

http://dx.doi.org/10.1016/j.jsams.2007.02.013

Castagna, C.; Impellizzeri, F. M.; Chaouachi, A.; Ben Abdelkrim, N., \& Manzi, V. (2011). Physiological responses to ball-drills in regional level male basketball players. Journal of Sports Science, 29(12), 1329-1336.

http://dx.doi.org/10.1080/02640414.2011.597418

Cohen, J. (1988). Statistical power analysis for the behavioural sciences. New York: Academic Press.

Coutts, K. D. (1998). Heart rates of participants in wheelchair sports. Paraplegia, 26, 4349.

http://dx.doi.org/10.1038/sc.1988.9

Croft, L., Dybrus, S., Lenton, J., \& Goosey-Tolfrey, V. (2010). A comparison of the physiological demands of wheelchair basketball and wheelchair tennis. International Journal of Sports Physiology and Performance, 5(3), 301-15. 
Yanci, J.; Iturricastillo, A.; Lozano, L.; Granados, C. (2015). Análisis de la condición física de jugadores nacionales de baloncesto en silla atendiendo a la clasificación funcional. RICYDE. Revista internacional de ciencias del deporte, 40(11), 173-185. http://dx.doi.org/10.5232/ricyde2015.04006

De Groot, S.; Balvers, I. J.; Kouwenhoven, S. M., \& Janssen T. W. (2012). Validity and reliability of tests determining performance-related components of wheelchair basketball. Journal of Sports Science, 30(9), 879-887.

http://dx.doi.org/10.1080/02640414.2012.675082

De Lira, C. A. B.; Vancini, R. L.; Minozzo, F. C.; Sousa, B. S.; Dubas, J. P.; Andrade, M. S.; Steinberg, L.L., \& Da Silva, A. C. (2010). Relationship between aerobic and anaerobic parameters and functional classification in wheelchair basketball players. Scandinavian Journal of Medicine and Science in Sports, 20(4), 638-643.

http://dx.doi.org/10.1111/j.1600-0838.2009.00934.x

Erikssen, G. (2001). Physical fitness and changes in mortality: the survival of the fittest. Sports Medicine, 31(8), 571-576.

http://dx.doi.org/10.2165/00007256-200131080-00001

Foster, C.; Florhaug, J. A.; Franklin, J.; Gottschall, L.; Hrovatin, L.A.; Parker, S.; Doleshal, P., \& Dodge C. (2001). A new approach to monitoring exercise training. Journal of Strength and Conditioning Research, 15(1), 109-115.

Gonaus, C., \& Müller, E. (2012). Using physiological data to predict future career progression in 14- to 17-year-old Austrian soccer academy players. Journal of Sports Science, 30(15), 1673-82.

http://dx.doi.org/10.1080/02640414.2012.713980

Goosey-Tolfrey, V. (2005). Physiological profiles of elite wheelchair basketball players in preparation for the 2000 paralympic games. Adapted Physical Activity Quarterly, 22(1), 57-66.

Goosey-Tolfrey, V., \& Tolfrey, K. (2008). The multi-stage fitness test as a predictor of endurance fitness in wheelchair athletes. Journal of Sports Sciences, 26(5), 511-517. http://dx.doi.org/10.1080/02640410701624531

Gorostiaga, E.; Izquierdo, M.; Ruesta, M.; Iribarren, J.; González-Badillo, J. J., \& Ibáñez, J. (2004). Strength training effects on physical performance and serum hormones in soccer players. European Journal of Applied Physiology, 91, 698-707.

http://dx.doi.org/10.1007/s00421-003-1032-y

Granger, D. A.; Schwartz, E. B.; Booth, A., \& Arentz, M. (1999). Salivary testosterone determination in studies of child health and development. Hormonal Behavior, 35, 8-27. http://dx.doi.org/10.1006/hbeh.1998.1492

Hellison, D. (2003). Training responsibility through physical activity (2nd ed.). Champaign, IL: Human Kinetics.

Hutzler, Y.; Chacham-Guber, A., \& Reiter, S. (2013). Psychosocial effects of reverseintegrated basketball activity compared to separate and no physical activity in young people with physical disability. Research in Developmental Disabilities, 34(1), 579-587. http://dx.doi.org/10.1016/j.ridd.2012.09.010

IWBF. (2010). Manual de clasificación del jugador de baloncesto en silla de ruedas. Consultado el 9 de enero de 2015, disponible en:

http://www.iwbf.org/images/a_classification/20102014ClassificationManualSpanishVersion.pdf

Jaarsma, E. A.; Dijkstra, P. U.; Geertzen, J. H., \& Dekker, R. (2014). Barriers to and facilitators of sports participation for people with physical disabilities: A systematic review. Scandinavian Journal of Medicine and Science in Sports. [Epub ahead of print]. http://dx.doi.org/10.1111/sms.12218

Kolt, G. S.; Kirkby, R. J.; Bar-Eli, M.; Blumenstein, B.; Chadha, N. K., \& Liu, J. (1999). A cross-cultural investigation of reasons for participation in gymnastics. International Journal of Sport Psychology, 30, 381-398. 
Yanci, J.; Iturricastillo, A.; Lozano, L.; Granados, C. (2015). Análisis de la condición física de jugadores nacionales de baloncesto en silla atendiendo a la clasificación funcional. RICYDE. Revista internacional de ciencias del deporte, 40(11), 173-185. http://dx.doi.org/10.5232/ricyde2015.04006

Leicht, C. A.; Bishop, N.C., \& Goosey-Tolfrey, V. L. (2012). Submaximal exercise responses in tetraplegic, paraplegic and non spinal cord injured elite wheelchair athletes. Scandinavian Journal of Medicine and Science in Sports, 22(6), 729-736. http://dx.doi.org/10.1111/j.1600-0838.2011.01328.x

Longmuir, P. E., \& Bar-Or, O. (2000). Factors influencing the physical activity levels of youths with physical and sensory disabilities. Adapted Physical Activity Quarterly, 17, 4053.

Los Arcos, A.; Yanci, J.; Mendiguchia, J.; \& Gorostiaga, E. M. (2014). Rating of muscular and respiratory perceived exertion in professional soccer players. Journal of Strength and Conditioning Research, 28(11), 3280-3288.

http://dx.doi.org/10.1519/JSC.0000000000000540

McVeigh, S. A.; Hitzig, M. A.; \& Craven, B. C. (2009). Influence of sport participation on community integration and quality of life: A comparison between sport participants and non-sport participants with spinal cord injury. Journal of Spinal Cord Medicine, 32, 115124.

Molik, B.; Kosmol, A.; Morgulec, N.; Hübner-Woźniak, E., \& Rutkowska, I. (2006). Anaerobic performance in a Polish first league team of wheelchair basketball players. Research Yearbook, 12(2), 199-202.

Molik, B.; Laskin, J. J.; Kosmol, A.; Skucas, K., \& Bida U. (2010). Relationship between functional classification levels and anaerobic performance of wheelchair basketball athletes. Research Quarterly in Exercise and Sport, 81(1), 69-73. http://dx.doi.org/10.1080/02701367.2010.10599629

Molik, B.; Laskin, J.; Kosmol, A.; Marszalek, J.; Morgule-Adamowicz, N., \& Frick, T. (2013). Relationships between anaerobic performance, field tests, and level of elite female wheelchair basketball athletes. Human Movement, 14(4), 366-371. http://dx.doi.org/10.2478/humo-2013-0045

Myers, J.; Kaykha, A.; George, S.; Abella, J.; Zaheer, N.; Lear, S.; Yamazaki, T., \& Froelicher, V. (2004). Fitness versus physical activity patterns in predicting mortality in men. American Journal of Medicine, 117(12), 912-918.

http://dx.doi.org/10.1016/j.amjmed.2004.06.047

Nash, M. S.; Van de Ven, I.; Van Elk, N., \& Johnson, B. M. (2007). Effects of circuit resistance training on fitness attributes and upper-extremity pain in middle-aged men with paraplegia. Archives of Physical Medicine and Rehabilitation, 88, 70-75. http://dx.doi.org/10.1016/j.apmr.2006.10.003

Pentland, W. E., \& Twomey, L. T. (1994). Upper limb function in persons with long term paraplegia and implications for independence: Part I. Paraplegia, 32, 211-218. http://dx.doi.org/10.1038/sc.1994.40

Peterson, M. J.; Giuliani, C.; Morey, M. C.; Pieper, C. F.; Evenson, K. R.; Mercer, V.; Cohen, H. J.; Visser, M.; Brach, J. S.; Kritchevsky, S. B.; Goodpaster, B. H.; Rubin, S.; Satterfield, S.; Newman, A. B., \& Simonsick, E.M. (2009). Physical activity as a preventative factor for frailty: the Health, Aging, and Body Composition Study. Journals of Gerontology Series A: Biological Sciences and Medical Sciences, 64(1), 61-68. http://dx.doi.org/10.1093/gerona/gln001

Plinta, R.; Saulicz, E.; Gnat, R.; Juras, G., \& Waskiewicz, Z. (2005). Assessment of specific coordination in subjects with handicapped locomotor system. Journal of Human Kinetics, 14, 41-50.

Rimmer, J. H. (2001). Physical fitness levels of persons with cerebral palsy. Developmental Medicine and Child Neurology, 43, 208-212.

http://dx.doi.org/10.1111/j.1469-8749.2001.tb00189.x 
Yanci, J.; Iturricastillo, A.; Lozano, L.; Granados, C. (2015). Análisis de la condición física de jugadores nacionales de baloncesto en silla atendiendo a la clasificación funcional. RICYDE. Revista internacional de ciencias del deporte, 40(11), 173-185. http://dx.doi.org/10.5232/ricyde2015.04006

Sassi, R. H.; Dardouri, W.; Yahmed, M. H.; Gmada, N.; Mahfoudhi, M.E., \& Gharbi, Z. (2009). Relative and absolute reliability of a Modified Agility T-Test and its relationship with vertical jump and straight sprint. Journal of Strength and Conditioning Research, 23(6), 1644-1651.

http://dx.doi.org/10.1519/JSC.0b013e3181b425d2

Shephard, R. (1995). Physical activity fitness and health: The current consensus. Quest, 47 (3), 288-303.

http://dx.doi.org/10.1080/00336297.1995.10484158

Sherrill, C. (2004). Adapted physical activity, recreation and sport: Cross disciplinary and lifespan (6th ed). Dubuque, IA: Brown \& Benchmark.

Van der Woude, L. H.; De Groot, S., \& Janssen, T. W. (2006). Manual wheelchairs: Research and innovation in rehabilitation, sports, daily life and health. Medical Engineering and Physics, 28(9), 905-915.

http://dx.doi.org/10.1016/j.medengphy.2005.12.001

Vanlandewijck, Y. C.; Daly, D. J., \& Theisen, D. M. (1999). Field test evaluation of aerobic, anaerobic, and wheelchair basketball skill performances. International Journal of Sports Medicine, 20, 548-554.

http://dx.doi.org/10.1055/s-1999-9465

Vanlandewijck, Y. C.; Evaggelinou, C.; Daly, D. J.; Verellen, J.; Van Houtte, S.; Aspeslagh, V.; Hendrickx, R.; Piessens, T., \& Zwakhoven, B. (2004). The relationship between functional potential and field performance in elite female wheelchair basketball players. Journal of Sports Science, 22(7), 668-675.

http://dx.doi.org/10.1080/02640410310001655750

Wang, Y. T.; Chen, S.; Limroongreungrat, W., \& Change, L. S. (2005). Contributions of selected fundamental factors to wheelchair basketball performance. Medicine and Science in Sports and Exercise, 37, 130-137.

http://dx.doi.org/10.1249/01.MSS.0000150076.36706.B2

Yanci, J.; Reina, R.; Los Arcos, A., \& Cámara, J. (2013). Effects of different contextual interference training programs on agility and speed performance of primary school students. Journal of Sports Science and Medicine, 12, 601-607.

Yanci, J.; Granados, C.; Otero, M.; Badiola, A.; Olasagasti, J.; Bidaurrazaga, I.; Iturricastillo, A., \& Gil, S. M. (2015). Sprint, agility, strength and endurance capacity in wheelchair basketball players. Biology of Sport, 32, 71-77.

http://dx.doi.org/10.5604/20831862.1127285 